\title{
Dislocations in the melting of two-dimensional electron crystals
}

To cite this article: B Tanatar and S -T Chui 1994 J. Phys.: Condens. Matter 6 L485

\section{Related content}

\begin{tabular}{l} 
- Observation of Dislocations in the Monte \\
$\frac{\text { Carlo Simulation of Flux Lattice Melting in }}{\text { High-T Superconductors }}$ \\
\hline S. T. Chui \\
- Topological Defects, Multiparticle \\
$\frac{\text { Exchange and Re-entrant Melting }}{\text { S. T. Chui and Xiu Qiu }}$ \\
- Quantum melting of a two-dimensional \\
$\frac{\text { Wigner crystal }}{\text { V T Dolgopolov }}$
\end{tabular}

View the article online for updates and enhancements.

\section{Recent citations \\ Emission limited electrostatic atomization and the fine structure constant A. J. Kelly \\ A.J. Kelly \\ Polaron in the Wigner lattice}

\section{IOP ebooks'}

Bringing you innovative digital publishing with leading voices to create your essential collection of books in STEM research. Start exploring the collection - download the first chapter of every title for free. 


\title{
LETTER TO THE EDITOR
}

\section{Dislocations in the melting of two-dimensional electron crystals}

\author{
B Tanatari and S-T Chuif \\ $\dagger$ Department of Physics, Bilkent University, Bilkent, 06533 Ankara, Turkey \\ $\ddagger$ Bartol Research Institute, University of Delaware, Newark, DE 19716, USA
}

Received 16 June 1994

\begin{abstract}
We study the defect structure of quantum Monte Carlo simulations of a twodimensional electron gas in the crystalline phase. A finite density of dislocation pairs exists even before the melting takes place. The dislocation pair density in the solid increases gradually as the electron gas parameter $r_{\mathrm{s}}$ is increased and is much higher than that in the related problem of flux-line lattice melting.
\end{abstract}

There has been much interest in the low-density limit of a two-dimensional (2D) electron gas. This provides a theoretical model to understand the many-body physics of several important applications. The pioneering ideas for the behaviour of low-density electron systems were put forward by Wigner. According to his argument the electrons may form a solid of lattice constant $a_{0}$ (also $n=1 / \pi a_{0}^{2}$ where $n$ is the areal density of electrons) at low densities due to the interplay between the kinetic energy cost to localize an electron and the Coulomb energy. Defining the dimensionless electron gas parameter $r_{\mathrm{s}}=a_{0} / a_{\mathrm{B}}$ where $a_{\mathrm{B}}=\hbar^{2} \epsilon / m e^{2}$ is the Bohr radius, one can show that the kinetic and potential energies are proportional to $1 / r_{\mathrm{s}}^{2}$ and $1 / r_{\mathrm{s}}$, respectively. Thus at low densities (large $r_{\mathrm{s}}$ ) the potential energy dominates, favouring the crystal phase, and at high densities (small $r_{\mathrm{s}}$ ) the kinetic energy determines the liquid phase. The experimental observation of the melting of a $2 \mathrm{D}$ electron crystal is achieved in the classical regime for a layer of electrons on the surface of liquid $\mathrm{He}$ [1]. The melting of quantum crystals is studied for a $2 \mathrm{D}$ electron gas in GaAs heterostructures and Si MOSFETs under a strong magnetic field and in Si MOSFETs in zero field [2].

The variational [3] and diffusion Monte Carlo [4] simulations of a 2D electron system were performed to investigate the ground-state properties at $T=0$. A solid-to-liquid melting transition is found [4] to occur at $r_{s}=37 \pm 5$ by comparing the energy of the two phases. Here we repeat similar Monte Carlo (MC) calculations to study the role played by defects in the melting process. In the diffusion MC calculation, the time development of the product of the 'exact' wave function and a variational wave function is studied. In this letter, this variational wave function is taken to be appropriate for the solid phase, which consists of a product of a Slater determinant of Gaussian orbitals located at lattice sites and a Jastrow-type correlation factor. This is what is meant by saying we are 'in the solid' phase in the following.

Chui and Esfarjani [5] have proposed a mechanism of spontaneous generation of dislocations for the melting of a quantum solid in $2 \mathrm{D}$ at zero temperature. Their predicted melting density agrees very well with MC simulations [4] and experimental results [6]. They also proposed that a bound dislocation pair provided for activated longitudinal transport [5], 
non-activated Hall transport [7], and non-linear transport that can depend on the external electric field to the $\frac{3}{2}$ power [7]; all in reasonable agreement with experiment. In a related system of flux-line lattices a similar melting mechanism was proposed [8] and the defect structures of MC simulations were analysed [9] to test these ideas.

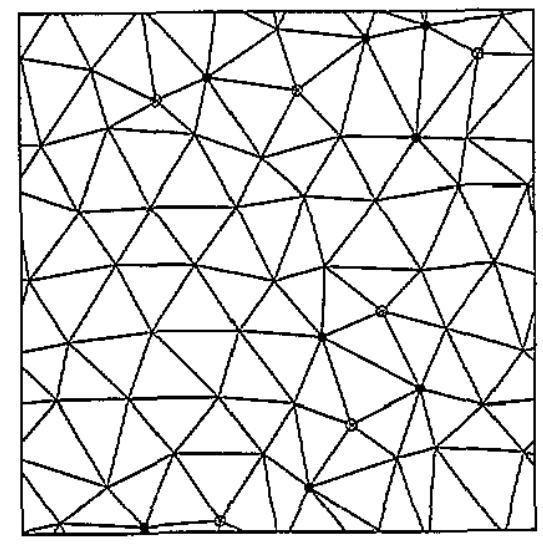

(a) $r_{3}=40 \quad N=56$

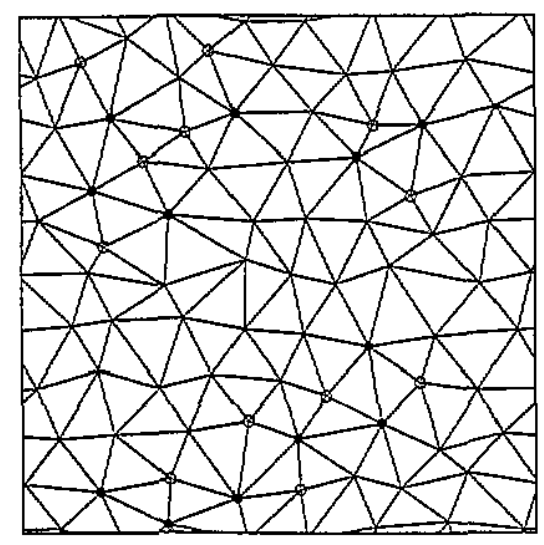

(c) $I_{\mathrm{g}}=75 \mathrm{~N}=80$

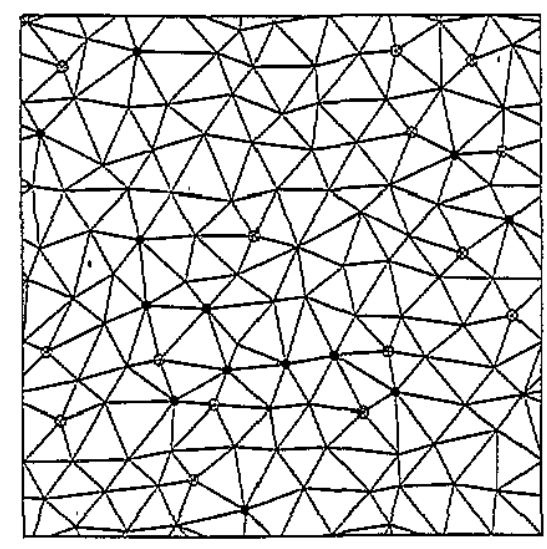

(b) $r_{3}=50 \quad N=120$

Figure 1. Delaunay triangulations of positions of electrons for typical $M C$-simulation-generated configurations. The electron density and systems sizes are such that (a) $r_{s}=40$, $N=56$; (b) $r_{s}=50, N=120$; (c) $r_{s}=75, N=80$. Filled (open) circles indicate particles with seven (five) neighbours.

Intrinsic defects occur naturaily in ordinary solids due to thermal fluctuations. Quantum fluctuations can also induce the formation of defects. In order to demonstrate the importance of dislocations, we analyse the configurations generated in the MC simulations of a $2 \mathrm{D}$ electron gas in terms of Delaunay triangulations [10]. The Delaunay triangulation is the problem of joining $N$ points in the plane by non-intersecting straight lines so that every region inside the boundary of the points is itself a triangle [10,11]. In figure 1 we depict typical configurations for the 2D electron crystal at various $r_{\mathrm{s}}$ values. Particles with seven neighbours are identified by filled circles, whereas those with five neighbours are identified by open circles. A declination in these 2D systems is represented as a particle with either five or seven nearest neighbours. A dislocation, on the other hand, is identified as a bound dislocation pair, with five or seven neighbours next to each other. Figure 1(a) shows a 
configuration at $r_{\mathrm{s}}=40$, which is close to the melting point (melting occurs at $r_{\mathrm{s}}=37 \pm 5$ for a pure system [4]) for an $N=56$-particle system. Figure 1(b) shows a configuration at a lower density $\left(r_{s}=50\right)$, when the $N=120$-particle electron system is in the crystalline phase. We observe that a finite number of bound dislocation pairs exist before the melting takes place. At even lower densities $\left(r_{\mathrm{s}}=75\right)$, the dislocations remain as shown in figure $1(\mathrm{c})$. These observations from the configurations of $\mathrm{MC}$ simulations of a $2 \mathrm{D}$ electron gas are in contrast with what is obtained for flux-line lattices.

In figure 2 we show the average density of defects as a function of electron density in units of the melting density. The data were collected for $\sim 1000$ steps per particle in the diffusion MC after an initial run of $\sim 500$ steps per particle in the variational MC. The average density of disclinations having five nearest neighbours is given in figure 2(a) for various lattice sizes. As we have seen directly from the typical configurations above, the defect density decreases gradually as $r_{s}^{*} / r_{\mathrm{s}}$ is decreased $\left(r_{s}^{*}=37\right.$ denotes the melting density), largely independent of the system size. Also shown in the same figure (the solid line with large error bars) is the corresponding defect density for the flux-line lattice [9] as a function of temperature in units of the melting temperature. The qualitative difference between the two cases is evident. As the density of disclinations vanishes below the melting point for the flux-line system, it remains finite for the electron system. Figure 2(b) shows the average density of disclinations with seven nearest neighbours, yielding similar behaviour. The defect densities in figure 2 are calculated from a large number of configurations generated during the MC runs, and the error bars indicate the statistical fluctuations. The number of fives and the number of sevens are not always equal to each other. An example of this is seen in the upper right hand corner of figure 1(a). We do not completely understand this result. There are several possible explanations. (i) In a finite-size system, the strain energy involved in creating isolated disclination at some finite time interval is finite. (ii) The local disturbance may be large enough that the interpretation in terms of Delaunay triangulation and disclinations may not be adequate. (iii) Image effects and the vector nature of the defect conspire to effect a net cancellation of the net strain.

Applying periodic boundary conditions to our finite-size simulation boxes, we calculate the average distance between a pair of observed disclinations. Figure 3 displays $\left\langle d_{\text {pairs }}\right\rangle$ as a function of the electron gas parameter $r_{\mathrm{s}}$ for different-size systems. In all cases examined the average size of the dislocation pair (or the average distance between two disclinations) gradually decreases as the melting point $\left(r_{\mathrm{s}} \sim 40\right)$ is crossed. The curves in figure 3 are obtained from the results of MC simulations for electrons in the crystal phase.

Our data do not indicate appreciable separations between the dislocation pairs in the solid phase. We suspect that there is a finite barrier for the pairs to separate, thus making it difficult to observe the separation with the simulation time that we have used.

The finite density of closely bound dislocation pairs in the ground state can come about because of the non-zero overlap between the phonon and dislocation wave functions. We have recently studied the physics of dislocation waves [12] and found that the wave function $|c\rangle$ of a dislocation pair located at $c_{1}, c_{2}$ can be approximated by the groundstate phonon wave function $|G\rangle$ but with the electrons displaced by static displacements $u_{k}^{0}\left[\exp \left(\mathrm{i} k c_{1}\right)-\exp \left(\mathrm{i} k c_{2}\right)\right]$ due to the intrinsic defect

$$
\begin{aligned}
& |G\rangle=\prod_{k} \mathrm{e}^{\left(-b_{k}\left|\zeta_{k}\right|^{2}\right)} /\left[\prod_{k}\left(\pi / b_{k}\right)^{0.25}\right] \\
& |c\rangle=\prod_{k} \mathrm{e}^{\left(-b_{k}\left|\xi_{k}+u_{k}^{0}\right|^{2}\right)} /\left[\prod_{k}\left(\pi / b_{k}\right)^{0.25}\right]
\end{aligned}
$$



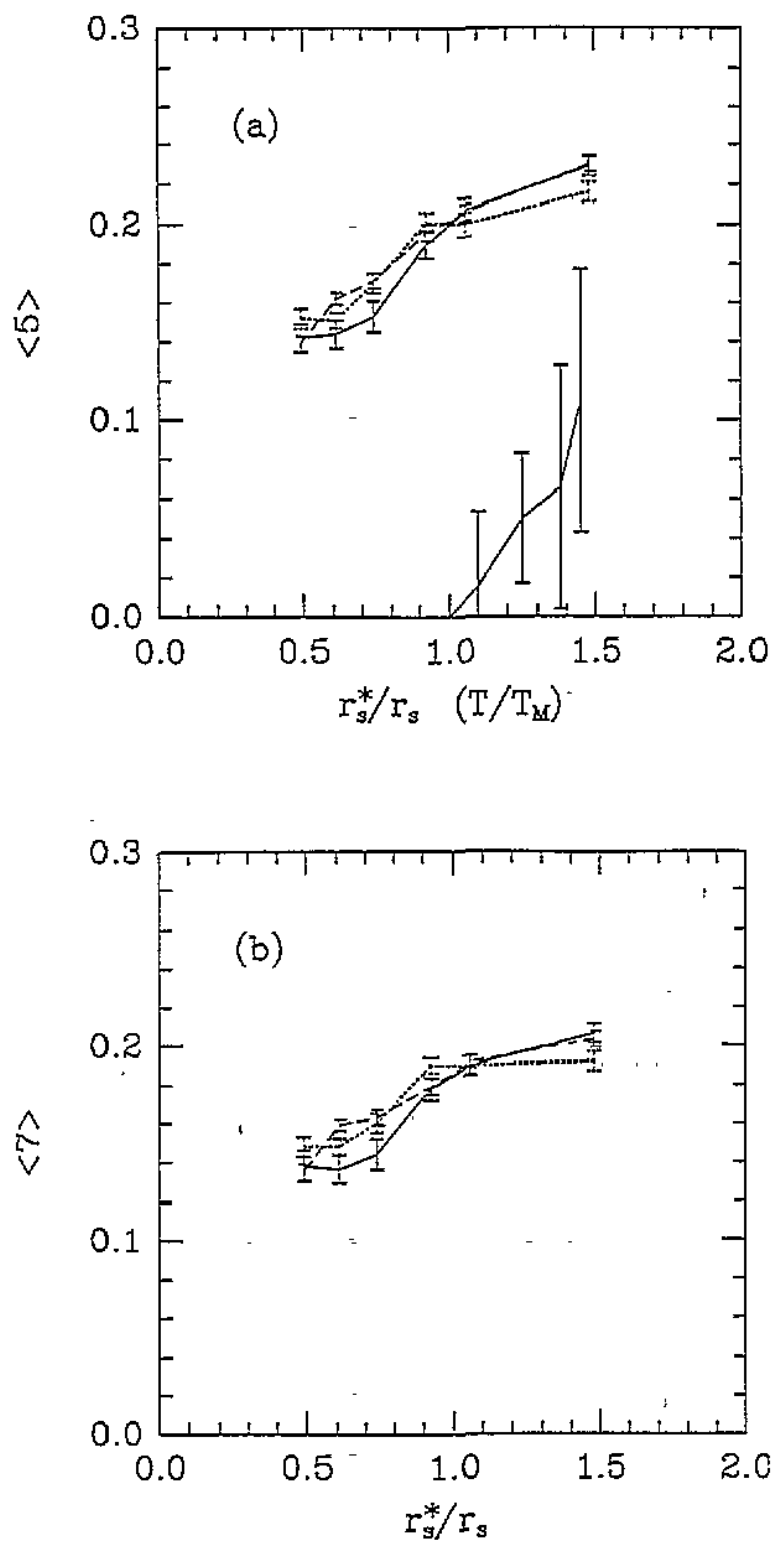

Figure 2. (a) The average density of five-neighbour declinations as a function of $r_{s}$ scaled with the melting point $r_{s}^{*}=37$. Solid, dotted, and dashed lines are for $N=56-, 80$-, and 120-particle systems, respectively. The error bars are due to statistical averaging over many configurations. Also shown, by a solid line with large error bars, is the defect density for a flux-line lattice as a function of temperature in units of melting temperature. (b) As (a) for the seven-neighbour disclination density.

where $b_{k} \simeq m \omega_{k} / 2 . \quad u_{k}^{0}$ is the Fourier transform of the static displacement due to a dislocation at the origin. We estimate the overlap between $|c\rangle$ and $|G\rangle$ to be $\langle G \mid c\rangle \simeq$ $\exp \left[-\sum_{i} w_{i} \delta c_{i}^{2}\right]$, where $w_{i}=\sum_{k} b_{k}\left|u_{k}^{0}\right|^{2} k_{i}^{2}$. To illustrate the numerical value of $w$, we take $\delta c_{y}$ to be zero and the Burger vector to be along the $x$ direction. $w_{x} \simeq 6.4 \times 10^{-2} \sqrt{r_{\mathrm{s}}} / a_{0}^{2}$, which is significant only if the dislocations are separated so that $\delta c_{x} \simeq a_{0}$ and $\delta c_{y}=0$. 


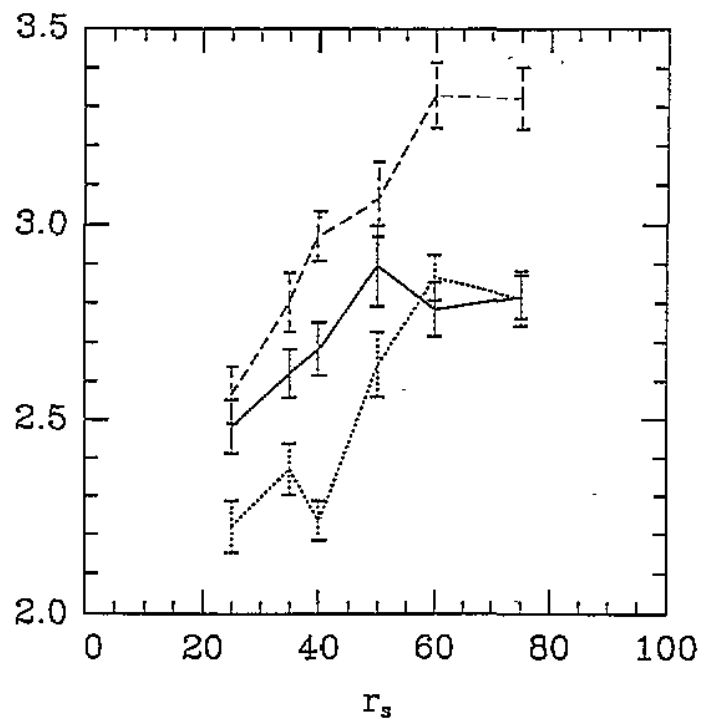

Figure 3. The average distance between five-neighbour and seven-neighbour disclination pairs as a function of $r_{\mathrm{s}}$. Solid, dotted, and dashed lines are for $N=56-, 80-$, and 120-particle systems, respectively, with the error bars originating from statistical averaging over many configurations.

This significant overlap, coupled with the fact that the net core energy of a dislocation pair is rather small, provides for a significant density of closely bound dislocation pairs. The data presented here indicate the presence of defects at different time slices only and are not a direct proof of an eigenstate, however.

The finite density of dislocation pairs lends support to the importance of dislocation pairs in the solid and its possible implications for the transport properties [5,7]. Because we are in the solid phase, the present result does not provide for direct evidence of the melting mechanisms of Chui and Esfarjani [5].

We now discuss briefly the similarities and differences with flux-line lattice melting, and the nature of the melting transition for $2 \mathrm{D}$ electron systems: The core energy in the flux-line lattices [13], which includes the thermal fluctuation effects, is substantial at the melting point. The corresponding dislocation core energy for the electron system is much smaller. We think the difference between the core energies gives rise to the distinguishing characteristics in the defect density. For small core energies, the system favours the generation of dislocations, which form grain boundaries, whereas for large core energies the unbinding of dislocation pairs becomes more advantageous. The smallness of the core energy for a $2 \mathrm{D}$ electron system gives some clues to the understanding of the nature of melting transition. Although classical melting [14] of the Wigner crystal at high temperature is found to be a continuous transition, MC simulations [15] record hysteresis effects and suggest a first-order transition. The transition from continuous to discontinuous behaviour was found [16] to occur at $E_{\mathrm{c}} / k_{\mathrm{B}} T_{\mathrm{KT}}=2.84$, where $T_{\mathrm{KT}}$ is the Kosterlitz-Thouless temperature. In the case of classical electrons $E_{\mathrm{c}} / k_{\mathrm{B}} T_{\mathrm{KT}}=4.8$ at $r_{\mathrm{s}}=37$, thus a continuous transition is expected. In the quantum limit, the dislocation wave function has a wider spread, which reduces the core energy by an order of magnitude. This leads us to believe the transition to be discontinuous. In contrast to the finite-temperature case, there can be long-range order at zero temperature in $2 \mathrm{D}$. A transition with a weak discontinuity is indicated in a recent experiment [17]. 
This work was supported by a NATO collaborative research grant (CRG-920487). BT thanks the Bartol Research Institute for hospitality during his stay in Newark where part of this work was done.

\section{References}

[1] Grimes C C and Adams G 1979 Phys. Rev. Lett. 42795 Gallet F, Deville G, Valdes A and Williams F I B 1982 Phys. Rev. Lett. 9212

[2] For a review see Chui S-T (ed) 1994 Physics of the 2D Quantum Electron Solid (Hong Kong: International) Willett R L, Stormer H L and Tsui D C 1988 Phys. Rev. B 387881

Andrei E Y, Deville G, Glattli D C, Williams F I B, Paris E and Etienne B 1988 Phys. Rev. Lett. 602765

[3] Ceperley D 1978 Phys. Rev. B 183126

[4] Tanatar B and Ceperley D M 1989 Phys. Rev. B 395005

[5] Chui S-T and Esfarjani K 1991 Europhys. Lett. 14 361; 1991 Phys. Rev. Lett. 66652

[6] Goldman V, Santos M, Shayegan M and Cunningham J 1990 Phys. Rev. Lett. 652189

[7] Chui S-T 1993 Solid State Commun. 86 605; 1993 Phys. Lett. 180A 149

[8] Ma H R and Chui S-T 1991 Phys. Rev. Lett. 67505

[9] Chui S-T 1992 Europhys. Lett. 20535

[10] Preparata F P and Shamos M I 1985 Computational Geometry (New York: Springer)

[11] We have used the program voroNol, which can be obtained from netlib@ornl.gov.

[12] Esfarjani K, Chui S-T and Qiu X 1992 Phys. Rev. B 464638

[13] Chui S-T and Ma H R 1992 J. Phys.: Condens. Matter 4 L237

[14] Glattli D C, Andrei E Y and Williams F I B 1988 Phys. Rev. Lett. 60420

[15] Imada M and Takahashi M 1984 J. Phys. Soc. Japan 533770

[16] Chui S-T 1983 Phys. Rev. B 28178

[17] Pudalov V and Chui S-T 1994 Phys. Rev. B 4914062 